\title{
Obstacle Avoidance System for UAVs using Computer Vision
}

\author{
John Dayton, Miguel Enriquez, Mathew Gan, James Liu, Jordan Quintana, and Blin Richards \\ California State Polytechnic University, Pomona, CA 91768
}

\begin{abstract}
The purpose of this research is to develop an obstacle avoidance system for use on small, fixed-wing Uninhabited Aerial Vehicles (UAVs). In order to detect and avoid obstacles, computer based vision algorithms will be implemented with an automatic flight control system. Images of obstacles are captured using forward facing, externally mounted cameras. Obstacles will include moving and non-moving objects within the flight path of the UAV, which will be detected through the use of optical flow and feature-tracking methods.
\end{abstract}

\section{Motivation and Goals for Research}

UAVs have the potential to replace inhabited aircraft for many civilian and military applications, which include, but not limited to, disaster relief assistance, search and rescue, and combat zone intelligence gathering. They have lower operating costs and pose minimal risk to human pilots. However, the lack of obstacle avoidance capabilities has limited the use of these vehicles. UAVs with inadequate sense and avoid capabilities are at risk of colliding with aerial objects, tall structures, and even trees. When collision takes place, damage to infrastructure and wildlife may occur, depending on the altitude and nature of the collision, in addition to the loss of the vehicle. Falling wreckage may pose even greater danger to building and humans underneath a UAV collision. For these reasons, current uses of UAVs are bound to military and restricted airspace. To be used in the National Airspace System (NAS), UAVs must comply with Federal Aviation Regulations requiring that they be equipped with obstacle avoidance systems.

In the recent past, research has been performed regarding the use of quadrotor UAVs. The advantages of quadrotors include their maneuverability and their ability to hover. However, they have lower limits 
regarding their payload capacity, speed, operational ceiling, and range, when compared to fixed-wing airframes. The capabilities of fixed-wing UAVs provide users with the options to carry out long-range forest, desert, and maritime search-and-rescue missions and battlefield reconnaissance. Due to the high speed requirements of fixed-wing aircraft, sonar technology prevalent on quadrotor UAVs can no longer be used, for the reason that travel time of sonar waves decreases the range that the UAV can detect obstacles. The alternative is optical cameras, which do not depend on propagating waves, but instead use light as its medium to detect objects. This way, the UAV can instantaneously detect images and can avoid obstacles much sooner.

Presently, Cal Poly Pomona is beginning development on an obstacle avoidance system for fixedwing UAVs. The intention is to create this system while using commercially available and low-cost components with the capacity for expansion. The rest of this paper will cover hardware selection and implementation, engineering and software approaches used, results thus far, and conclusion with future plans.

\section{Hardware}

In order to maintain a relatively inexpensive obstacle avoidance system components were selected on the criteria of being reliable and cost effective. All components selected are commercially available off the shelf to ensure the above criteria. Table 2.1 lists all components of the system. Each component as follows were selected base cost and performance primarily. The Ardupilot is one of the most inexpensive fully functioning autopilots available and it performs as well as most autopilots far more expensive. The PX4 Pixhawk autopilot is also as cost effective as the Ardupilot and is marketed to be more powerful, but it is a new system and previous experience with the Ardupilot made it the best choice. The Arduino UNO was chosen due to its simplicity and cost, the processing power of the micro controller was not a factor due to the simple operations the micro controller would be preforming. The Super-Vision camera was chosen due to its small size, video quality and ability to adapt to light changes. The Panda board was chosen for its size, cost and previous experience using it for image processing. The Sig Kadet Senior air frame was selected for its payload capacity and stable characteristics. Finally the JR 
$12 \mathrm{X}$ was chosen for its reliability and range, in order to maintain the safest conditions while the plane is in flight. Figure 2.1 shows the system architecture and how information will flow through the avoidance system.

\section{Methodology}

The overall system functionality is broken into three parts: autonomous flight, object recognition, and avoidance commands. While in autonomous flight, the aircraft is under the control of the ArduPilot Mega 2.5. Also during flight, the camera is collecting images and sending them to the onboard image processor, which filters and analyzes the images. If the processor detects an object in the images, it will begin to track it. As objects begin moving towards or are already in the flight path of the UAV, they are immediately tracked and upgraded to obstacles. When this happens, the processor sends an avoidance command to the microcontroller, which in turn will calculate the amount of control surface deflection required to move the UAV away from the obstacle.

To utilize computer-based vision for obstacle avoidance, a live video stream from a camera is required. An algorithm for obstacle avoidance was developed through an open source programming library, OpenCV. The primary function of the algorithm created in OpenCV is to detect and track an object. For this purpose, the computer-based vision algorithm utilizes two methods working simultaneously to obtain points of interest from the camera's live video stream: motion detection (optical flow) and feature detection, which are contained in the OpenCV libraries. Optical flow is used to detect, track, and predict the location of an object's motion in a video stream while feature detection is used to detect and track an object's feature, such as a corner or edge.

Feature detection defines and tracks primary points of interest of an image. This is done through the use of a corner detection method known as Shi-Tomasi, which is replicated in OpenCV's function goodFeaturesToTrack. The corner detection method uses minimum eigenvalues that act as threshold values for the quality level of a corner. If a corner is below the quality threshold value, that corner is not tracked. The function assigns a threshold value for the quality of the feature and the number of features to track in each frame of a video stream. Before using the function, certain criteria must be known: the 
number of corners to track, the quality of the corners, and the minimum distance between each corners. Figure 3.1, taken from OpenCV's goodFeaturesToTrack tutorial, shows an example of the implementation of feature detection, presenting 25 corners with a quality of 0 and a distance of 0.1 pixel units. Once these points of interest have been defined, the optical flows of those features are calculated through OpenCV's optical flow function.

Optical Flow, in short, is motion estimation. It is utilized in object tracking through feature comparisons of two frames. A feature found in the first frame is compared to the same feature found in the second frame. The displacement vector created by an object's specific pixel location (feature) from one point in a frame to another point in a different frame is what is known as the optical flow of that object. This type of optical flow in the computer-based vision algorithm is called the Lucas-Kanade method, and is represented by the OpenCV function calcOpticalFlowPyrLK. Utilizing the time difference between the two frames, velocity can be calculated to represent the motion.

Due to noise from the camera in feature tracking from one frame to another, Lucas-Kanade can only be used for small displacements. To solve this limitation, a method called the pyramids approach is used to calculate the Lucas-Kanade optical flow for larger displacements, by downscaling an image's resolution to make it smaller. This results in a smaller pixel displacement. Figure 3.2 shows the downscaling of an image and the decrease in pixel displacement by a factor of two. By downscaling an image's displacement and resizing the small displacement back to the image's original resolution, the Lucas-Kanade method can calculate large displacements.

By utilizing the Shi-Tomasi method to track a feature, and the Lucas-Kanade method with pyramids to track a moving object, a computer-based vision algorithm can be used to avoid both, static and dynamic, obstacles.

Although the ArduPilot firmware is open source and can be edited, in the interest of keeping the required computer coding to its simplest form, it was not modified to incorporate the avoidance commands. Instead, a secondary microcontroller sends the avoidance commands to the ArduPilot via pulse-width-modulation (PWM), similar to the manual remote control inputs that the ArduPilot receives 
from the safety pilot. This allows the autopilot to perform its intended function with no extra processing or interruptions that may hinder its ability to control the UAV. After the image processor has identified obstacles in the aircrafts path, a probability matrix is created that calculates the safest region of the image to fly. From this information, an avoidance command is sent via serial communication to the Arduino Uno, which then relays the command to the ArduPilot via PWM. The ArduPilot can be programmed to limit the response of the aircraft such as not allowing the plane to bank more than 30 degrees. These limits will be programmed in order to keep the plane from over correcting from the given avoidance inputs. After the obstacle has been successfully avoided, the autopilot will resume the flight path and continue its mission.

\section{Conclusion}

All three individual systems have been tested and are working, with the aircraft and autopilot having been flown and tuned for autonomous navigation. The computer vision code has also been tested on manual flights as seen in Figure 4.1, while the avoidance algorithm has successfully sent commands from a laptop computer to the Ardupilot Mega 2.5. Full-system ground tests, with single moving and stationary targets, have been conducted and the aircraft's attempted maneuvers and control surface deflections are as anticipated. More ground tests, such as moving objects from multiple directions, and multiple stationary objects, are required to ensure the system will respond appropriately in all circumstances. Once the system has been thoroughly evaluated flight test will begin. This project has laid the ground work for a more robust and comprehensive obstacle avoidance systems and will continue to be built upon in the years to come. 
Table 2.1: List of system components

\begin{tabular}{|c|c|c|}
\hline Component & Name & Cost \\
\hline Autopilot & Ardupilot Mega 2.5 & $\sim \$ 300$ \\
\hline Flight Controller & Arduino Uno & $\sim \$ 25$ \\
\hline Camera & Super-Vision CCD & $\sim \$ 140$ \\
\hline Image Processor & Panda Board ES & $\sim \$ 200$ \\
\hline Signal Management & 8 Channel Relay & $\sim \$ 10$ \\
\hline Airframe & Sig Kadet Senior & $\sim \$ 300$ \\
\hline Safety Control & JR 12X & $\sim \$ 1200$ \\
\hline
\end{tabular}

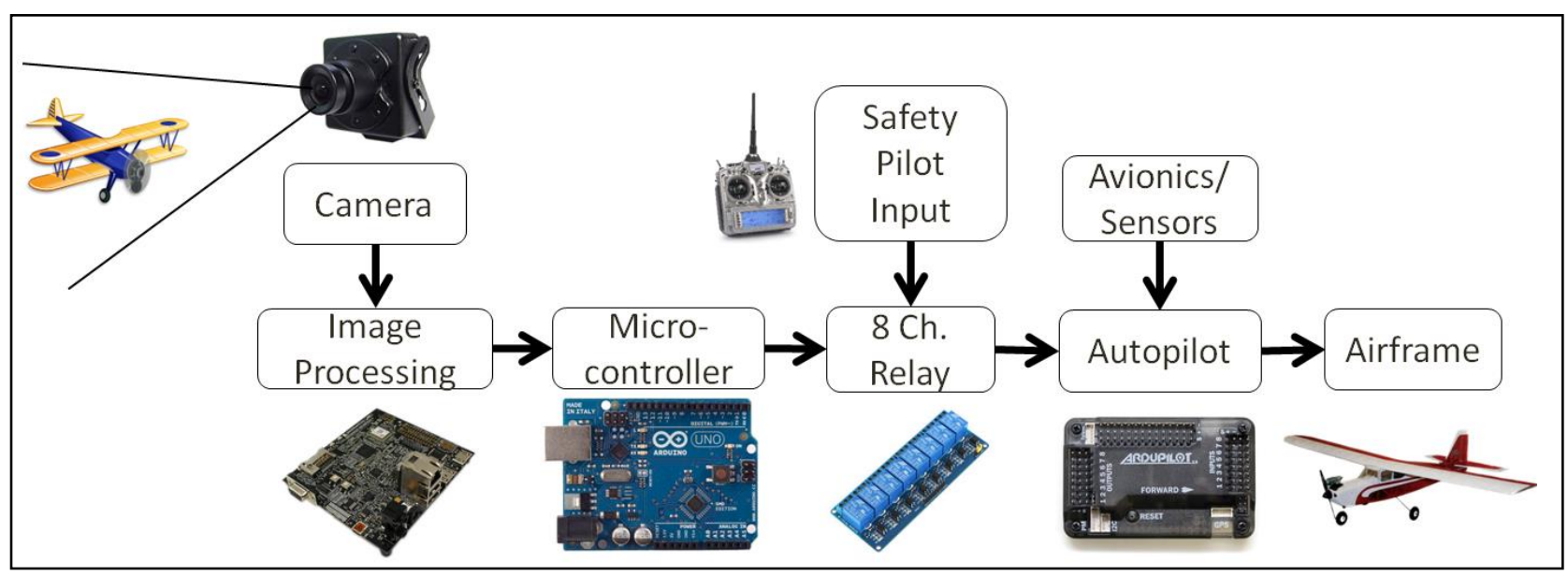

Figure 2.1: System Architecture

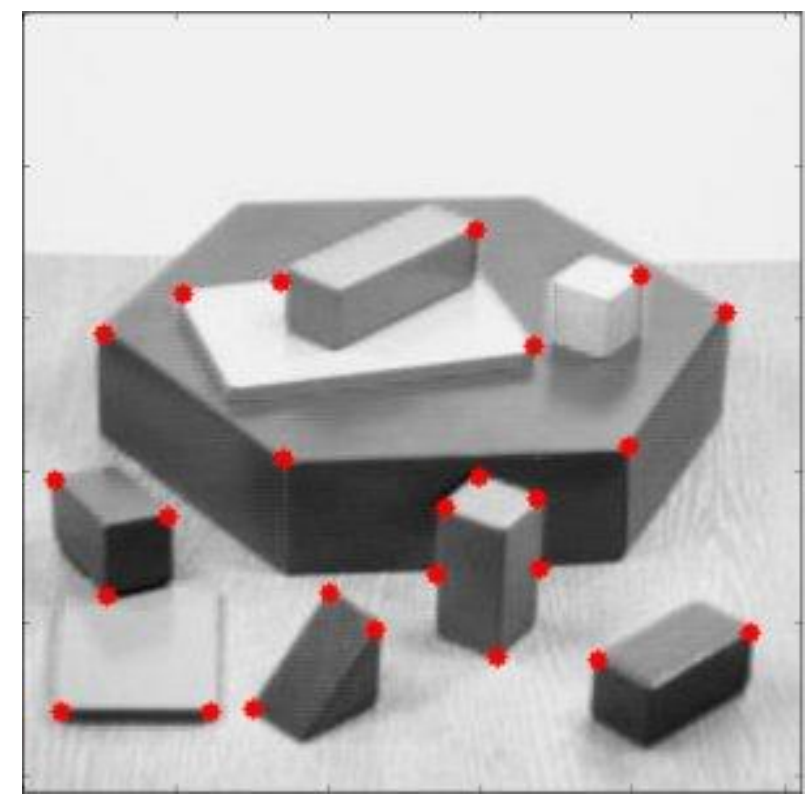

Figure 3.1: goodFeaturesToTrack Function 


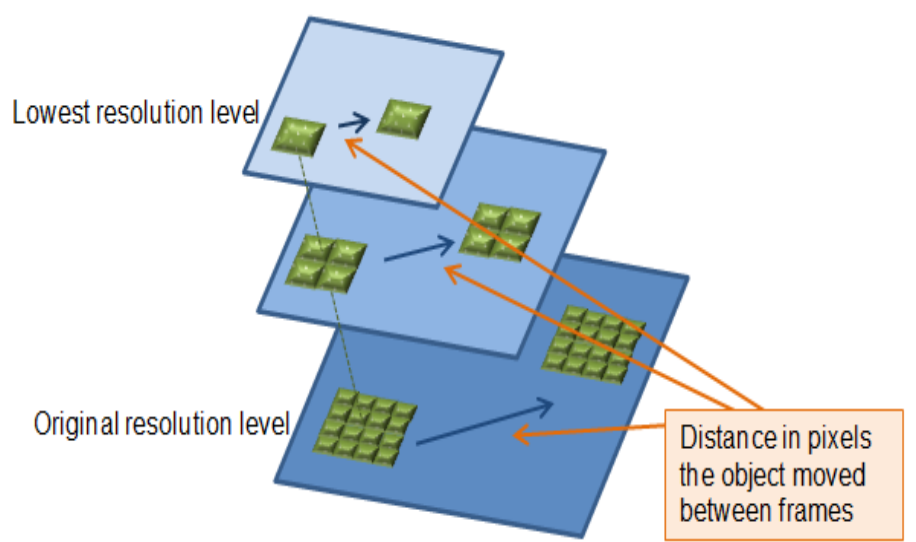

Pyramid Levels

Figure 3.2: Lucas-Kanade with Pyramids Application

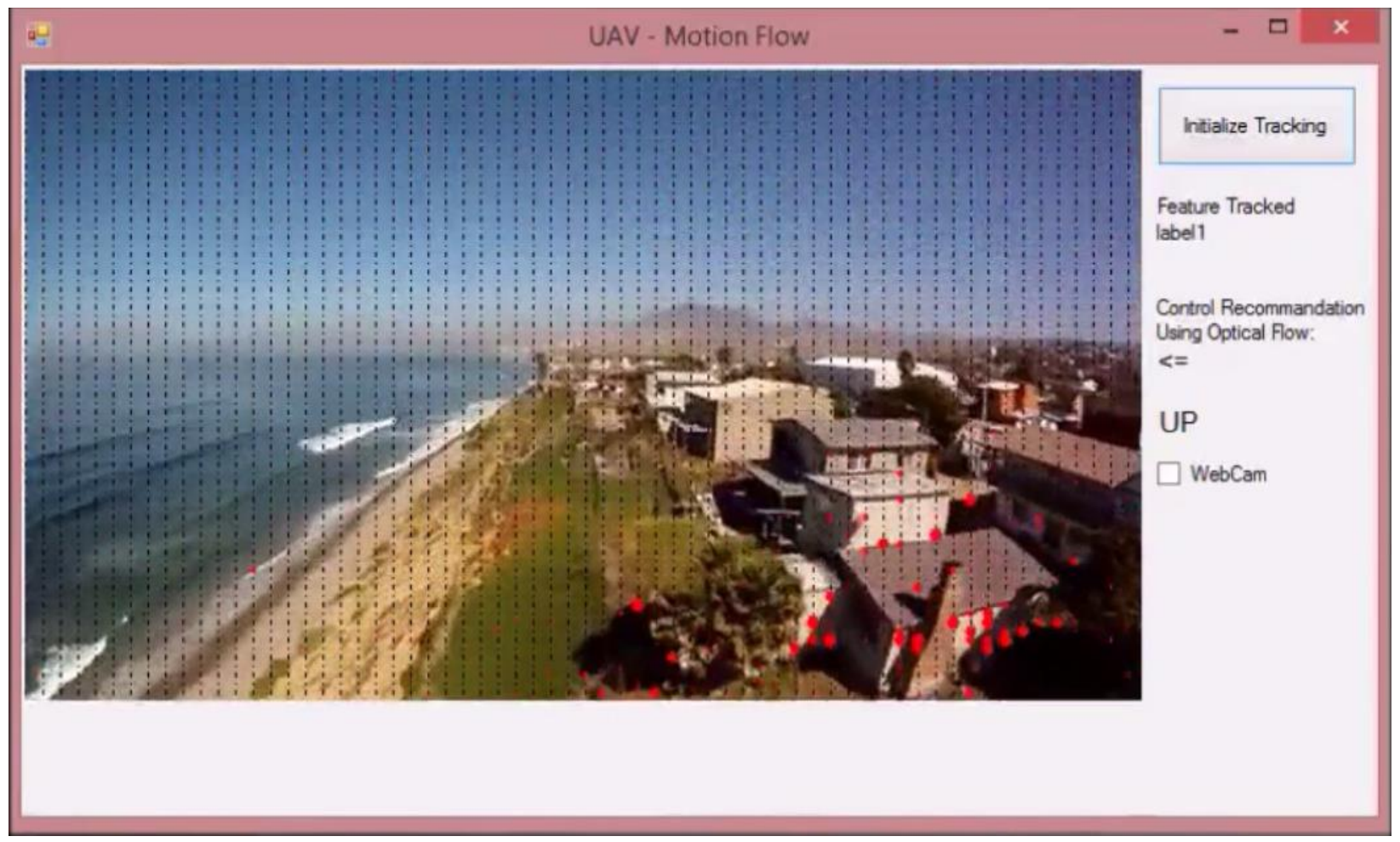

Figure 4.1: Example of computer vision on sample video 


\section{References}

[1] He, Z., Iyer, R. V., et al, "Vision-Based UAV Flight Control and Obstacle Avoidance", Proceedings of American Control Conference, Minneapolis, Minnesota, June, 14-16, 2006.

[2] Call, R., Brandon, "Obstacle Avoidance for Unmanned Aerial Vehicles”, Thesis for Bringham Young University, Idaho, United States, December 2006.

[3] DIY Drones, "Arduplane", https://code.google.com/p/ardupilot-mega/wiki/home?tm=6

[4] OpenCV Pyramid tutorial, http://docs.opencv.org/trunk/doc/py_tutorials/py_feature2d/py_shi_tomasi/py_shi_tomasi.ht $\underline{\mathrm{ml}}$

[5] Computer Vision Introduction, http://www.comp.nus.edu.sg/ cs4243/lecture/motion.pdf

[6] Vision Class Reference, http://www.mathworks.com/help/vision/ref/vision.pointtrackerclass.html

[7] Arduino Help and References, http://arduino.cc/en/Reference/HomePage\#.UyaNEfldXwE

\section{Special Thanks}

Subodh Bhandari, PhD: Professor, Aerospace Engineering Department, Cal Poly Pomona Bruce Wu: Computer Science Department, OpenCV help 